\title{
Cognitive Decline Predicts Long-Term Care Insurance Requirement Certification in Community-Dwelling Older Japanese Adults: A Prospective Cohort Study
}

\author{
Shu Nishiguchia, b Minoru Yamada ${ }^{a}$ Takuya Sonodac Hiroki Kayamaa

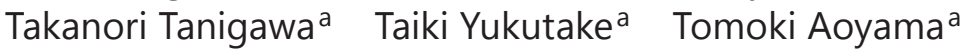 \\ a Department of Physical Therapy, Human Health Sciences, Graduate School of Medicine, \\ Kyoto University, Kyoto, b Japan Society for the Promotion of Science, Tokyo, and ' ${ }^{\mathrm{D}}$ Division of \\ Health Affairs Policy, Department of Health and Welfare of the Kyoto Prefecture, Kyoto, Japan
}

\author{
Key Words \\ Cognitive decline · Long-term care insurance requirement · Older Japanese adults . \\ Prospective cohort study
}

\begin{abstract}
Aim: The purpose of this prospective cohort study is to examine whether cognitive decline is an independent predictor of new long-term care insurance (LTCI) requirement certifications in Japan. Methods: A total of 5,765 community-dwelling older Japanese adults who, at baseline, were independent in terms of their activities of daily living participated in this study and were followed up for 18 months. The outcome measure was the number of new LTCI requirement certifications during the 18-month period of the study. We collected demographic information through questionnaires and assessed cognitive skills with the Cognitive Performance Scale (CPS). The participants were divided into 3 groups according to CPS scores $(0,1$, and 2 or greater). Results: During the 18-month period, 399 subjects (6.9\%) became newly certified for LTCI services. In a multivariate Cox proportional hazards model, older participants with a CPS score of 1 (adjusted HR: 1.39, 95\% CI: 1.08-1.77) and 2 or greater (adjusted HR: 2.27, 95\% CI: 1.74-2.96) were significantly more likely to receive an LTCI certification compared to those with a CPS score of 0 . Conclusions: Cognitive decline is an independent predictor of new LTCI requirement certifications and the severity of cognitive decline in elderly adults is positively associated with receiving an LTCI requirement certification in Japan.
\end{abstract}




\section{Introduction}

Frailty in older adults is a serious problem in countries with increasingly aging populations, such as Japan. In general, frailty is defined as a vulnerable state that places older adults at a high risk for adverse health outcomes, such as falls, hospitalization, and mortality [1].

To help manage an extremely rapidly aging population and care for the frail properly, in April 2000 Japan implemented a long-term care insurance (LTCI) system. Before 2000, long-term care services were provided under a tax-based social welfare system for senior citizens with limited economic resources and family support [2]. However, after the LTCI implementation, the services of this program have been provided to older adults who are certified as requiring support or care according to the certification assessment of their care needs [3]. The selection process for classifying dependent older adults involves evaluations of the persons' current mental and physical condition and objective assessments [4]. Thus, those who are certified as requiring LTCI services have variable risk factors associated with frailty.

A survey by the Japanese Ministry of Health, Labour and Welfare revealed that dementia is one of the main reasons for which people require LTCI services. Dementia affects 5-8\% of the population over 65 years of age [5] and up to $30 \%$ of people aged 85 years and above [6], and its prevalence is currently increasing. It is clear that dementia and Alzheimer's dementia (AD) are associated with mortality [7]. A previous study reported a positive association between physical frailty and cognitive impairment [8]. Furthermore, physical frailty has been associated with a risk for mild cognitive impairment (MCI) and a rapid rate of cognitive decline with age [9]. It has also been shown that cognitive decline is associated with frailty in Japan, and it is one of the predictors of frailty leading to the need for long-term care [10]. However, no studies have determined whether cognitive decline can be an independent predictor of new LTCI requirement certifications.

To fill this gap, the current prospective cohort study examines whether a new LTCI requirement certification can be predicted by using cognitive performance measures. We focused on whether the level of cognitive decline is related to receiving a new LTCI requirement certification among community-dwelling elderly Japanese people.

\section{Materials and Methods}

\section{Subjects}

We analyzed a portion of the cohort data from a prospective study, the Japan Multicenter Aging Cohort for Care Prevention (J-MACC). This cohort study investigated the factors associated with the need for LTCI services in community-dwelling Japanese adults aged 65 years or older living in Maibara City, Shiga Prefecture. In 2011, the total population was 39,889, the total area $250.46 \mathrm{~km}^{2}$, and the rate of the aging population $25.3 \%$. We recruited 8,233 community-dwelling older adults who were independent in their activities of daily living (ADL), and the baseline questionnaires were mailed to participants in April 2011 (response rate of $96 \%$ ). These questionnaires were mainly self-administered. However, if the participants could not answer questions independently, a family member assisted them or answered the questions on their behalf. Older adults who were already ADL-dependent and already receiving benefits from LTCI services were excluded. Furthermore, in the database, we excluded older adults who had missing baseline questionnaire data. Therefore, our analysis was performed with data from 5,765 older adults who were then followed up for 18 months. This study was conducted in accordance with the guidelines of the Declaration of Helsinki, 
and the study protocol was reviewed and approved by the Ethics Committee of the Kyoto University Graduate School of Medicine.

\section{Cognitive Performance}

We assessed cognitive performance by using the Cognitive Performance Scale (CPS) [11]. The CPS is based on a decision tree algorithm that includes items assessing daily decisionmaking, short-term memory, ability to make oneself understood, and ability to feed oneself. Cognitive decline is rated on a scale from 0 to $6(0=$ normal, $1=$ borderline intact, $2=$ mild, $3=$ moderate, $4=$ moderate-severe, $5=$ severe, and $6=$ very severe) [11]. The CPS is positively associated with the Mini-Mental State Examination (MMSE) and the Montreal Cognitive Assessment, which are well-known tools for the detection of cognitive impairment and MCI, respectively $[12,13]$. In the present study, we divided participants into 3 groups according to their CPS scores $(0=$ normal, $1=$ borderline intact, and 2 or greater $=$ cognitive impairment $)$, which have been shown to be highly correlated with MMSE scores in previous studies, thus indicating high convergent validity $[12,14]$.

\section{Outcome Measurement}

The outcome measure was the receipt of an LTCI requirement certification during the 18-month study period. Dependent older adults are classified according to the following procedure: first, a 74-item questionnaire is administered to evaluate the person's current mental and physical condition, and responses are analyzed using a computerized algorithm [4]. Subsequently, the person receives a home visit and a recommendation from his or her physician. Based on the questionnaire and home visit data, a long-term care approval board makes the final decision. Individuals who become certified as 'dependent older adults' are subdivided into seven levels (support levels 1 and 2, and care levels 1-5) based on their physical condition. Certified adults are provided home services and community-based or institutional services according to their care needs. Individuals who are not eligible for longterm care or support care may utilize preventive care services. In the present study, the city government recorded the date when individuals had been certified as meeting the LTCI requirements, and we updated our database as appropriate.

\section{Covariate Measurement}

In the baseline questionnaires, we obtained demographic information on age, gender, body mass index (BMI), the number of medications used (none, 1, 2, 3, or more), family structure (living alone, living in an elderly household, or other), subjective household economic status (very good, good, fair, or bad), and medical history (musculoskeletal disorder, hypertension, lipid disorder, stroke, diabetes, heart disease, respiratory disease, external injury, and cancer).

\section{Statistical Analysis}

The baseline characteristics of the participants who were newly certified or not certified as requiring LTCI services were compared using a Student $t$ test or a $\chi^{2}$ test. In addition, the differences in demographic variables among the 3 groups stratified by CPS score were examined using ANOVA with a post hoc test or a $\chi^{2}$ test.

Kaplan-Meier survival curves were calculated for the 3 groups of participants newly certified as requiring LTCI services. Using the Cox proportional hazards model, the hazard ratio (HR) and the $95 \%$ confidence interval (CI) were estimated to evaluate, in univariate and multivariate analyses, the impact of cognitive decline on the time of receiving an LTCI requirement certification. In the multivariate analysis, the results were adjusted for age, gender, BMI, medication use, family structure, subjective household economy, and medical 
Table 1. Baseline demographic differences in the LTCI service requirement certification during the 18-month follow-up

\begin{tabular}{|c|c|c|c|}
\hline & $\begin{array}{l}\text { LTCI requirement } \\
\text { certification } \\
(n=399)\end{array}$ & $\begin{array}{l}\text { No LTCI requirement } \\
\text { certification } \\
(n=5,366)\end{array}$ & $\mathrm{p}$ \\
\hline Age, years ${ }^{\mathrm{a}}$ & $80.7 \pm 6.9$ & $74.2 \pm 6.6$ & $<0.001^{* *}$ \\
\hline Female gender ${ }^{b}$ & $237(59.4)$ & $2,829(52.7)$ & $0.011^{*}$ \\
\hline $\mathrm{BMI}^{\mathrm{a}}$ & $22.2 \pm 3.5$ & $22.7 \pm 3.1$ & $0.012^{*}$ \\
\hline CPS score ${ }^{b}$ & & & $<0.001^{* *}$ \\
\hline 0 & $232(58.1)$ & $4,091(76.2)$ & \\
\hline 1 & $89(22.3)$ & $942(17.6)$ & \\
\hline 2 or greater & $78(19.5)$ & $333(6.2)$ & \\
\hline Number of medications taken ${ }^{b}$ & & & $<0.001^{* *}$ \\
\hline 0 & $41(10.3)$ & $1,021(19.0)$ & \\
\hline 1 & $35(8.8)$ & $809(15.1)$ & \\
\hline 2 & $59(14.8)$ & $943(17.6)$ & \\
\hline 3 or more & $264(66.2)$ & $2,593(48.3)$ & \\
\hline Family structure ${ }^{b}$ & & & $<0.001^{* *}$ \\
\hline Single & $70(17.5)$ & $491(9.2)$ & \\
\hline Elderly household & $73(18.3)$ & $1,521(28.3)$ & \\
\hline Other & $256(64.2)$ & $3,354(62.5)$ & \\
\hline Subjective household economic status ${ }^{\mathrm{b}}$ & & & 0.475 \\
\hline Very good & $22(5.5)$ & $218(4.1)$ & \\
\hline Good & $141(35.3)$ & $2,022(37.7)$ & \\
\hline Fair & $167(41.9)$ & $2,228(41.5)$ & \\
\hline Bad & $69(17.3)$ & $898(16.7)$ & \\
\hline \multicolumn{4}{|l|}{ Medical history ${ }^{\mathrm{b}}$} \\
\hline Musculoskeletal disorder & $88(22.1)$ & $685(12.8)$ & $<0.001^{* *}$ \\
\hline Hypertension & $170(42.6)$ & $2,452(45.7)$ & 0.252 \\
\hline Lipid disorder & $22(5.5)$ & $429(8.0)$ & 0.082 \\
\hline Stroke & $25(6.3)$ & $202(3.8)$ & $0.022^{*}$ \\
\hline Diabetes & $40(10.0)$ & $650(12.1)$ & 0.231 \\
\hline Heart disease & $80(20.1)$ & $702(13.1)$ & $<0.001^{* *}$ \\
\hline Respiratory disease & $37(9.3)$ & $251(4.7)$ & $<0.001^{* *}$ \\
\hline External injury & $18(4.5)$ & $158(2.9)$ & 0.095 \\
\hline Cancer & $25(6.3)$ & 207 (3.9) & $0.024^{*}$ \\
\hline
\end{tabular}

Values are mean \pm SD or $\mathrm{n}(\%) .{ }^{*} \mathrm{p}<0.05 ;{ }^{* *} \mathrm{p}<0.01 .{ }^{\mathrm{a}}$ Assessed by Student's t test. ${ }^{\mathrm{b}}$ Assessed by $\chi^{2}$ test.

history. Survival time was defined as the time between enrollment (the baseline measurements) and either time of receiving an LTCI service requirement certification or the end of the follow-up period (October 2012).

Statistical analyses were carried out using SPSS version 20.0 (SPSS, Chicago, Ill., USA), with a significance threshold of 0.05 .

\section{Results}

\section{Demographic Data}

During the 18 months of the study, 399 subjects (6.9\%) became newly certified as requiring LTCI services (table 1). Those who were certified for LTCI requirement were significantly older $(80.7 \pm 6.9$ vs. $74.2 \pm 6.6$ years, $p<0.001)$ and had a lower BMI $(22.2 \pm 3.5$ vs. 
Nishiguchi et al.: Cognitive Decline Predicts Long-Term Care Insurance Requirement Certification in Community-Dwelling Older Japanese Adults

Table 2. Baseline demographic differences according to CPS scores

\begin{tabular}{|c|c|c|c|c|c|}
\hline & \multicolumn{5}{|l|}{ CPS score } \\
\hline & $\begin{array}{l}0 \\
(n=4,323)\end{array}$ & $\begin{array}{l}1 \\
(n=1,031)\end{array}$ & $\begin{array}{l}2 \text { or greater } \\
(n=411)\end{array}$ & $\begin{array}{l}\mathrm{p} \text { value } \\
\text { for trend }\end{array}$ & $\begin{array}{l}\text { post hoc } \\
\text { test }\end{array}$ \\
\hline Age, years ${ }^{\mathrm{a}}$ & $74.1 \pm 6.6$ & $75.3 \pm 7.1$ & $77.9 \pm 7.3$ & $<0.001^{* *}$ & $\mathrm{c}-\mathrm{e}$ \\
\hline Female gender ${ }^{b}$ & $2,374(54.9)$ & $501(48.6)$ & $191(43.3)$ & $<0.001^{* *}$ & \\
\hline $\mathrm{BMI}^{\mathrm{a}}$ & $22.7 \pm 3.1$ & $22.4 \pm 3.2$ & $22.2 \pm 3.3$ & $0.001^{* *}$ & $c, e$ \\
\hline Medications $^{\mathrm{b}}$ & & & & $<0.001^{* *}$ & \\
\hline 0 & $853(19.7)$ & $154(14.9)$ & $55(12.5)$ & & \\
\hline 1 & $675(15.6)$ & $141(13.7)$ & $28(6.3)$ & & \\
\hline 2 & $792(18.3)$ & 156 (15.1) & $54(12.2)$ & & \\
\hline 3 or more & $2,003(46.3)$ & $580(56.3)$ & $274(62.1)$ & & \\
\hline Family structure ${ }^{b}$ & & & & $0.016^{*}$ & \\
\hline Single & $401(9.3)$ & $104(10.1)$ & $56(12.7)$ & & \\
\hline Elderly household & $1,216(28.1)$ & $298(28.9)$ & $80(18.1)$ & & \\
\hline Other & $2,706(62.6)$ & $629(61.0)$ & 275 (66.9) & & \\
\hline Subjective household economic status ${ }^{\mathrm{b}}$ & & & & $<0.001^{* *}$ & \\
\hline Very good & $664(15.4)$ & $190(18.4)$ & $113(25.6)$ & & \\
\hline Good & $1,765(40.8)$ & $460(44.6)$ & $170(38.5)$ & & \\
\hline Fair & $1,701(39.3)$ & $341(33.1)$ & $121(27.4)$ & & \\
\hline Bad & $193(4.5)$ & $40(3.9)$ & $7(1.6)$ & & \\
\hline \multicolumn{6}{|l|}{ Medical history ${ }^{b}$} \\
\hline Musculoskeletal disorder & $571(13.2)$ & $140(13.6)$ & $62(14.1)$ & 0.557 & \\
\hline Hypertension & $1,949(45.1)$ & $489(47.4)$ & $184(41.7)$ & 0.380 & \\
\hline Lipid disorder & $358(8.3)$ & $63(6.1)$ & $30(6.8)$ & 0.061 & \\
\hline Stroke & $138(3.2)$ & $43(4.2)$ & $46(10.4)$ & $<0.001^{* *}$ & \\
\hline Diabetes & $504(11.7)$ & $133(12.9)$ & $53(12.0)$ & 0.454 & \\
\hline Heart disease & $538(12.4)$ & $169(16.4)$ & $75(17.0)$ & $<0.001^{* *}$ & \\
\hline Respiratory disease & $183(4.2)$ & $63(6.1)$ & $42(9.5)$ & $<0.001^{* *}$ & \\
\hline External injury & $109(2.5)$ & $43(4.2)$ & $24(5.4)$ & $<0.001^{* *}$ & \\
\hline Cancer & $151(3.5)$ & $56(5.4)$ & $25(5.7)$ & $0.002^{* *}$ & \\
\hline
\end{tabular}

Values are mean \pm SD or $n(\%) .{ }^{*} \mathrm{p}<0.05 ;{ }^{* *} \mathrm{p}<0.01$. CPS score: 0 (normal), 1 (borderline intact), 2-6 (cognitive impairment). ${ }^{\text {a }}$ Assessed by ANOVA. ${ }^{\text {b }}$ Assessed by $\chi^{2}$ test. ${ }^{c}$ Significant difference between a CPS score of 0 and a CPS

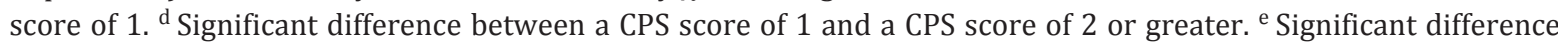
between a CPS score of 0 and a CPS score of 2 or greater.

$22.7 \pm 3.1, \mathrm{p}=0.012)$ than those who were not certified. More women than men became certified in this cohort (females: 59.4\%; males: $52.7 \%, p=0.011$ ). In addition, there were significant differences in the rates of high CPS scores, medication use, family structure, and some of the medical history items between the groups (table 1).

The baseline demographic data of the participants stratified into 3 groups according to CPS score are shown in table 2. The number of older adults with a CPS score of 0 was $4,323(75.0 \%)$, with a CPS score of 1 it was 1,031 (17.9\%), and with a CPS score of 2 or greater it was $411(7.1 \%)$. ANOVA showed that older adults with a CPS score of 0 were significantly younger (CPS score 0: $74.1 \pm 6.6$ years vs. CPS score 1: $75.3 \pm 7.1$ years vs. CPS score 2 or greater: $77.9 \pm 7.3$ years) and had a higher BMI (CPS score 0: $22.7 \pm 3.1$ vs. CPS score $1: 22.4 \pm 3.2$ vs. CPS score 2 or greater: $22.2 \pm 3.3$ ) than those with a CPS score of 1 or 2 or greater $(\mathrm{p}<0.01)$. In addition, there were significant differences in gender, medication use, family structure, household economy, and some of the medical history items between the 3 groups (table 2). 
Fig. 1. Kaplan-Meier survival curves illustrating the percentage of subjects who were not certified for LTCI services. During the study period, 232 (5.4\%), 89 (8.6\%), and $78(19.0 \%)$ subjects with CPS scores of 0,1 , and 2 or greater, respectively, became newly certified for LTCI services.

Table 3. Cox proportional hazards model for the impact of cognitive decline on the time of receiving an LTCI requirement certification
Nishiguchi et al.: Cognitive Decline Predicts Long-Term Care Insurance Requirement Certification in Community-Dwelling Older Japanese Adults

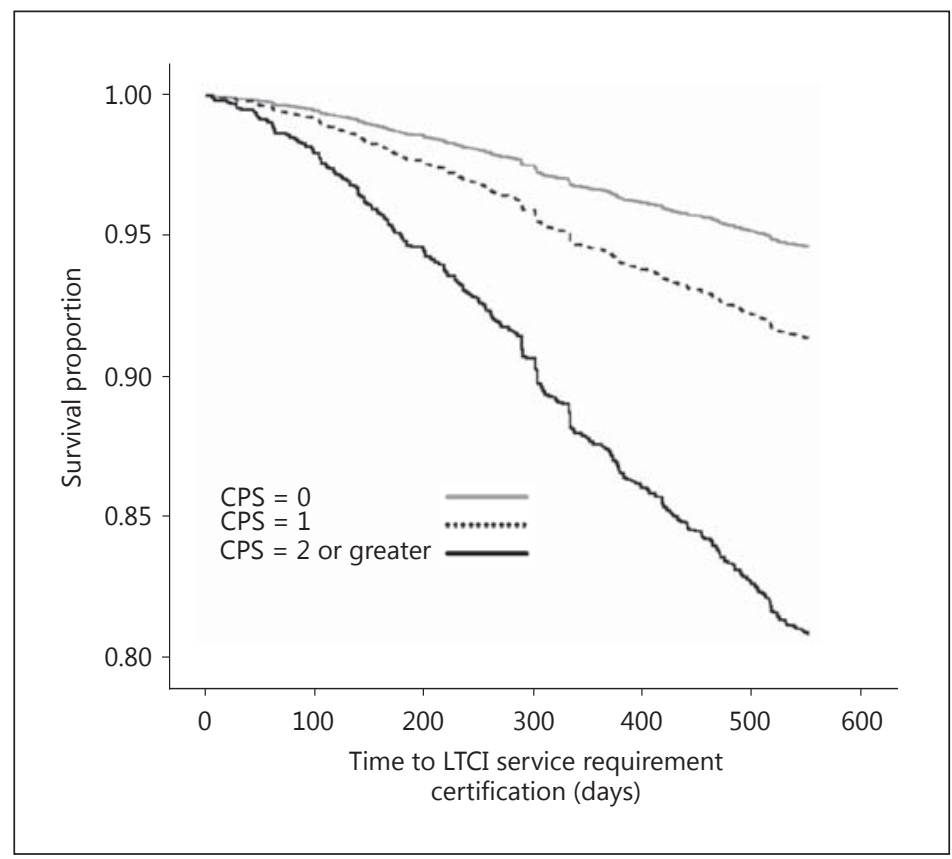

\begin{tabular}{|c|c|c|c|c|}
\hline \multirow{2}{*}{$\begin{array}{l}\text { CPS } \\
\text { score }\end{array}$} & \multicolumn{2}{|l|}{ Univariate model } & \multicolumn{2}{|c|}{ Multivariate model } \\
\hline & HR (95\% CI) & $\mathrm{p}$ & HR (95\% CI) & $\mathrm{p}$ \\
\hline 0 & 1 (ref.) & - & 1 (ref.) & - \\
\hline 1 & $1.64(1.28-2.09)$ & $<0.001$ & $1.39(1.08-1.77)$ & 0.009 \\
\hline $2-6$ & $3.85(2.98-4.98)$ & $<0.001$ & $2.27(1.74-2.96)$ & $<0.001$ \\
\hline
\end{tabular}

CPS score: 0 (normal), 1 (borderline intact), 2-6 (cognitive impairment). The multivariate model was adjusted for age, gender, BMI, number of medications taken, family structure, subjective household economy, and past medical history.

\section{Cox Proportional Hazards Model}

Figure 1 shows the Kaplan-Meier survival curves during the 18-month follow-up according to new LTCI service requirement certifications, with the participants stratified into 3 groups according to their CPS score. During the 18-month period, 232 (5.4\%), 89 (8.6\%), and $78(19.0 \%)$ subjects with CPS scores of 0,1 , and 2 or greater, respectively, became newly certified as requiring LTCI services.

Older participants with CPS scores of 1 (adjusted HR: 1.64, 95\% CI: 1.28-2.09) and 2 or greater (adjusted HR: 3.85, 95\% CI: 2.98-4.98) were significantly more likely to be certified as requiring LTCI services according to the univariate analyses using a CPS score of 0 as the reference (table 3). In multivariate analyses, these results remained significant after adjustment for age, gender, BMI, medication use, family structure, subjective household economy, and past medical history [adjusted HR (CPS score 1): 1.39, 95\% CI: 1.08-1.77; adjusted HR (CPS score 2 or greater): 2.27, 95\% CI: 1.74-2.96] (table 3). 


\section{Discussion}

In this study, we showed that cognitive decline was an independent predictor of new certifications for LTCI service requirement after adjusting for personal and social information. Cognitive decline is also associated with impairment of instrumental ADL [15] and gait dysfunction $[16,17]$ from its early stages. Furthermore, cognitive decline is a major risk factor for falls, which are a serious health problem among older adults with or without cognitive impairment [18]. Even MCI has been viewed as a predictor of falls [19]. Thus, cognitive decline is a risk factor for frailty in older adults, both directly and indirectly.

The current study indicates that a higher CPS score was associated with a greater possibility of certification for LTCI services. This finding is important from a clinical or research perspective. The cognitive assessment used in this study (CPS) consists of questions assessing various parts of subjective cognitive decline. According to several previous studies, this measure is closely associated with objective cognitive status evaluations in cross-sectional analysis [20] and predicts the development of dementia [21, 22]. Therefore, subjective cognitive decline based on the CPS is a valid risk factor for frailty in elderly populations. Furthermore, older participants with a CPS score of 1 may manifest MCI or very early dementia, and older participants with MCI may be more likely to be certified as requiring LTCI. Therefore, older adults should be targets of the preventive care system even before the first stages of cognitive impairment.

Recently, numerous research projects have investigated the impact of interventions designed to prevent the progression of cognitive impairment, such as $\mathrm{AD}$ [23]. It has been shown that regular exercise is associated with a delay in dementia and AD onset [24]. Furthermore, a multicomponent exercise program concentrating on aerobics was effective for improving cognitive function in older adults with amnestic MCI [25]. Thus, physical exercise can improve cognitive impairment and may be helpful in delaying or preventing the need for LTCI services. In Japan, community-based exercise programs have been found to have beneficial effects on frail older adults [26]. Hence, preventive programs should become widespread and exercise programs should be utilized by older adults.

This study has several limitations. First, we did not include older adults who had missing baseline questionnaire data. In this population, there may be older adults with more severe cognitive impairment or at a higher risk for frailty. Second, the questionnaires in the current study were primarily self-administered. However, if the participants could not answer questions independently, a family member assisted them or answered the questions on their behalf. We did not analyze the difference between those who could answer questionnaires by themselves and those who could not. The latter group may also have more severe cognitive impairment or a higher risk for frailty. Third, although we assessed subjective cognitive performance by using the questionnaire-based CPS, the participants' global objective cognitive function was not assessed by the MMSE or other objective measures.

In conclusion, our results indicate that cognitive decline is an independent predictor of new LTCI requirement certifications, and the severity of cognitive decline in the elderly is positively associated with receiving an LTCI requirement certification in Japan. Intervention studies are needed to explore whether improvement in cognitive impairment may delay or prevent the need for LTCI services among older adults.

\section{Acknowledgements}

This study was supported in part by Grants-in-Aid for Scientific Research from the Ministry of Education, Culture, Sports, Science and Technology of Japan. 


\section{Disclosure Statement}

The authors have no conflicts of interest to disclose.

\section{References}

-1 Fried LP, Tangen CM, Walston J, Newman AB, Hirsch C, Gottdiener J, Seeman T, Tracy R, Kop WJ, Burke G, McBurnie MA: Frailty in older adults: evidence for a phenotype. J Gerontol A Biol Sci Med Sci 2001;56:M146-M156.

-2 Campbell JC, Ikegami N: Long-term care insurance comes to Japan. Health Aff (Millwood) 2000;19:26-39.

-3 Tsutsui T, Muramatsu N: Care-needs certification in the long-term care insurance system of Japan. J Am Geriatr Soc 2005; 53:522-527.

-4 Tsutsui T, Muramatsu N: Japan's universal long-term care system reform of 2005: containing costs and realizing a vision. J Am Geriatr Soc 2007;55:1458-1463.

-5 Groves WC, Brandt J, Steinberg M, Warren A, Rosenblatt A, Baker A, Lyketsos CG: Vascular dementia and Alzheimer's disease: is there a difference? A comparison of symptoms by disease duration. J Neuropsychiatry Clin Neurosci 2000;12:305-315.

-6 Ferri CP, Prince M, Brayne C, Brodaty H, Fratiglioni L, Ganguli M, Hall K, Hasegawa K, Hendrie H, Huang Y, Jorm A, Mathers C, Menezes PR, Rimmer E, Scazufca M: Global prevalence of dementia: a delphi consensus study. Lancet 2005;366:2112-2117.

7 Todd S, Barr S, Roberts M, Passmore AP: Survival in dementia and predictors of mortality: a review. Int J Geriatr Psychiatry 2013, E-pub ahead of print.

8 Buchman AS, Boyle PA, Wilson RS, Tang Y, Bennett DA: Frailty is associated with incident Alzheimer's disease and cognitive decline in the elderly. Psychosom Med 2007;69:483-489.

-9 Boyle PA, Buchman AS, Wilson RS, Leurgans SE, Bennett DA: Physical frailty is associated with incident mild cognitive impairment in community-based older persons. J Am Geriatr Soc 2010;58:248-255.

10 Fukutomi E, Okumiya K, Wada T, Sakamoto R, Ishimoto Y, Kimura Y, Kasahara Y, Chen WL, Imai H, Fujisawa M, Otuka K, Matsubayashi K: Importance of cognitive assessment as part of the 'Kihon Checklist' developed by the Japanese Ministry of Health, Labor and Welfare for prediction of frailty at a 2-year follow up. Geriatr Gerontol Int 2013;13:654-662.

11 Morris JN, Fries BE, Mehr DR, Hawes C, Phillips C, Mor V, Lipsitz LA: MDS Cognitive Performance Scale. J Gerontol 1994;49:M174-M182.

12 Hartmaier SL, Sloane PD, Guess HA, Koch GG, Mitchell CM, Phillips CD: Validation of the Minimum Data Set Cognitive Performance Scale: agreement with the Mini-Mental State Examination. J Gerontol A Biol Sci Med Sci 1995;50:M128-M133.

13 Jones K, Perlman CM, Hirdes JP, Scott T: Screening cognitive performance with the Resident Assessment Instrument for Mental Health Cognitive Performance Scale. Can J Psychiatry 2010;55:736-740.

14 Büla CJ, Wietlisbach V: Use of the Cognitive Performance Scale (CPS) to detect cognitive impairment in the acute care setting: concurrent and predictive validity. Brain Res Bull 2009;80:173-178.

15 Umegaki H, Suzuki Y, Yanagawa M, Nonogaki Z, Nakashima H, Kuzuya M, Endo H: Cognitive impairments and functional declines in older adults at high risk for care needs. Geriatr Gerontol Int 2013;13:77-82.

16 Montero-Odasso M, Verghese J, Beauchet 0, Hausdorff JM: Gait and cognition: a complementary approach to understanding brain function and the risk of falling. J Am Geriatr Soc 2012;60:2127-2136.

17 Verghese J, Wang C, Lipton RB, Holtzer R, Xue X: Quantitative gait dysfunction and risk of cognitive decline and dementia. J Neurol Neurosurg Psychiatry 2007;78:929-935.

18 Ganz DA, Bao Y, Shekelle PG, Rubenstein LZ: Will my patient fall? JAMA 2007;297:77-86.

19 Delbaere K, Kochan NA, Close JC, Menant JC, Sturnieks DL, Brodaty H, Sachdev PS, Lord SR: Mild cognitive impairment as a predictor of falls in community-dwelling older people. Am J Geriatr Psychiatry 2012;20:845-853.

-20 Amariglio RE, Townsend MK, Grodstein F, Sperling RA, Rentz DM: Specific subjective memory complaints in older persons may indicate poor cognitive function. J Am Geriatr Soc 2011;59:1612-1617.

21 St John P, Montgomery P: Are cognitively intact seniors with subjective memory loss more likely to develop dementia? Int J Geriatr Psychiatry 2002;17:814-820.

22 Verdelho A, Madureira S, Moleiro C, Santos CO, Ferro JM, Erkinjuntti T, Poggesi A, Pantoni L, Fazekas F, Scheltens P, Waldemar G, Wallin A, Inzitari D: Self-perceived memory complaints predict progression to Alzheimer disease. The LADIS study. J Alzheimers Dis 2011;27:491-498. Ballard C, Gauthier S, Corbett A, Brayne C, Aarsland D, Jones E: Alzheimer's disease. Lancet 2011;377:1019-1031. Larson EB, Wang L, Bowen JD, McCormick WC, Teri L, Crane P, Kukull W: Exercise is associated with reduced risk for incident dementia among persons 65 years of age and older. Ann Intern Med 2006;144:73-81.

-25 Suzuki T, Shimada H, Makizako H, Doi T, Yoshida D, Tsutsumimoto K, Anan YA, Uemura K, Lee S, Park H: Effects of multicomponent exercise on cognitive function in older adults with amnestic mild cognitive impairment: a randomized controlled trial. BMC Neurol 2012;12:128.

26 Yamada M, Arai H, Sonoda T, Aoyama T: Community-based exercise program is cost-effective by preventing care and disability in Japanese frail older adults. J Am Med Dir Assoc 2012;13:507-511. 